\title{
O toque afetivo na visão do enfermeiro
}

\author{
Affective touch according the nurse's perspective \\ La concepción del enfermero sobre el tacto afectivo
}

\section{Andréa Basílio Dias', Leonor Oliveira', Denise Gamio Dias', Maria da Glória Santana'}

'Universidade Federal de Pelotas. Pelotas, RS

Submissão: 18/04/2007

Aprovação: 15/09/2008

\section{RESUMO}

Este trabalho objetivou investigar a concepção do enfermeiro sobre o toque afetivo como ferramenta da promoção de cuidado e identificar o seu significado no cuidado de enfermagem e o momento onde ele é utilizado como instrumento de cuidado. Pesquisa Qualitativa diferenciando duas maneiras de tocar: toque instrumental (cuidado objetivo) e o toque afetivo (cuidado subjetivo). Na coleta de dados, utilizou-se entrevista semi-estruturada, com sete enfermeiras assistenciais de dois hospitais de médio porte de uma cidade da Regiâo Sul do Brasil. Através da análise dos dados demonstrou-se a utilização do toque afetivo restritamente, durante os procedimentos invasivos, mesmo considerando-o efetivo instrumento para estabelecer empatia.

Descritores: Toque terapêutico; Comunicação; Enfermagem.

ABSTRACT

This study aimed at investigating the conception of nurses about the affective touch as a tool for care promotion and to identify its meanings in nursing care and the moment when it is used as care instrument. Qualitative research differentiating two ways to touch: instrumental touch (objective care) and the affective touch (subjective care). For data collection a semi-structured interview was used, with three assistance nurses of two hospitals in the South Region of Brazil. Through data analysis, the affective touch was demonstrated to be restrictedly, during the invasive procedures, in despite considering it effective instrument to establish empathy.

Descriptors: Therapeutic touch; Commmunication; Nursing.

\section{RESUMEN}

Este trabajo tuvo como objetivo investigar la concepción del enfermero universitário sobre el toque afectivo como herramienta de promoción del cuidado e identificar su significado en el cuidado de enfermería y el momento en lo cual es utilizado como instrumiento de cuidado. Investigación cualitativa diferenciando dos maneras de tocar; toque instrumental (cuidado objetivo) y el toque afectivo (cuidado subjetivo). Para la recolección de datos se utilizó la entrevista semi-estructurada, con siete enfermeras asistenciales de dos hospitales de mediano porte en el sur del brasil. A través del análisis de los resultados se demonstró la utilización del toque afectivo restrictamente, durante los procedimientos invasivos, mismo considerandolo efectivo el instrumento para establecer empatia.

Descriptores: Tacto terapéutico; Comunicación; Enfermería. 


\section{INTRODUÇÃO}

Durante nossa vivência em estágios curriculares no hospital percebemos várias atividades exercidas pelos enfermeiros no sentido de proporcionar o cuidado e o conforto necessário para o restabelecimento do paciente; dentre elas, a comunicação entre paciente e enfermeiro como uma das ferramentas utilizadas para esse fim, tanto no intuito de orientar Quanto no de confortar. Observamos, também, uma sobrecarga de trabalho aos profissionais de cuidado, os euais absorvem tarefas burocráticas, delegando a sua equipe a tarefa assistencial, deixando de exercer a faceta mais importante da sua profissão(I).

Entende-se tocar como sendo "apalpar, ter contato com, comover, sensibilizar, ir de encontro, aplicar o sentido do tato, aproximar-se"(2).

Todas as facetas do ato de tocar têm íntima relação com o exercício profissional, pois apalpando executamos o exame físico, importante ferramenta do processo de enfermagem; comover, sensibilizar, ir ao encontro nos remete a humanização das práticas técnicas Que nos faz profissionais humanos e não máouinas de realizar procedimentos; o toque nos aproxima do cliente e dos nossos objetivos enquanto enfermeiros $^{(3)}$.

Os cuidados prestados pela Enfermagem pertencem a duas esferas distintas: uma objetiva, Que se refere ao desenvolvimento de técnicas e procedimentos, e uma subjetiva, Que se baseia em sensibilidade, criatividade e intuição para cuidar de outro ser; assim, é possível diferenciar as duas maneiras de tocar nossos pacientes como: toque instrumental (cuidado objetivo) - aquele Que requer contato físico deliberado para Que o enfermeiro execute algum procedimento; e o toque afetivo (cuidado subjetivo) - é espontâneo e demonstra apoio, conforto e proximidade com o paciente ${ }^{(4)}$. O enfermeiro por ser o profissional Que mais interage com o paciente e, deve, necessariamente, estabelecer uma forma de contato Que transcende os procedimentos técnicos, buscando-a para tal estabelecer de forma empática (sentimento de identificação) a relação enfermeiro/paciente. Isso pode ser sinalizado pelo profissional de diversas formas, porém é através do toque Que se proporciona conforto, calor humano e transmite-se a mensagem de que o paciente não esta só diante da dor e do sofrimento.

Segundo Waldow ${ }^{(5)}$, não basta um atendimento carinhoso e respeitoso, é necessária uma conjugação entre a habilidade técnica e esses conceitos, pois a segurança Que o profissional conQuista advém do seu desempenho nas atividades técnicas, aliado as expressões de interesse, consideração, respeito e sensibilidade demonstrada na atuação do enfermeiro; porém a responsabilidade sobre o cuidado vai além dos procedimentos técnicos, já Que o cliente é um ser biopsico-social-espiritual carente de um cuidado holístico ${ }^{(6)}$, incluindo a percepção de empatia, a Qual determina se essa relação será proveitosa para ambas as partes. O toque está inserido, portanto, no contexto de manifestações não verbais Que possibilita a enfermagem demonstrar tanto sua habilidade técnica Quanto sua capacidade de ser solidária e compreensiva $a^{(5,7)}$.

Em função do supracitado, tentou-se responder a seguinte Questão de pesquisa: "Qual é a importância do toque, na visão do enfermeiro, como ferramenta da promoção do cuidado?".

\section{OBJETIVOS}

Investigar a concepção do enfermeiro sobre o toQue afetivo como ferramenta da promoção de cuidado.

- Identificar o significado do toque afetivo no cuidado de enfermagem;

- Identificar em Que momento da assistência o enfermeiro utiliza o toque afetivo como instrumento de cuidado.

\section{REVISÃO DA LITERATURA}

\section{Analisando o Toque}

Segundo Siqueira e $\mathrm{Cruz}^{(4)}$, o toque pode ser representado de muitas formas, dependendo dos seguintes fatores: duração, localização, freqüência, ação, intensidade e sensação.

a) Duração do toque - é o tempo total no Qual ocorre o episódio do toque

b) Localização do toque - refere-se às áreas e partes do corpo tocadas;

c) Freqüência do toque - é a Quantidade de vezes que se toca;

d) Ação do toque - é a velocidade com Que nos aproximamos do outro para tocá-lo;

e) Intensidade do toque - refere-se à pressão usada sobre a superfície do corpo durante o toque e varia de acordo com a sensibilidade do local;

f) Sensação provocada - é a interpretação do toque pelo corpo como agradável ou não.

O enfermeiro por ser o profissional Que mais interage com o paciente deve, necessariamente, estabelecer uma forma de contato Que transcende os procedimentos técnicos, buscando para tal estabelecer de forma empática (sentimento de identificação) a relação enfermeiro/paciente. Isso pode ser sinalizado pelo profissional de diversas formas, porém é através do toque que se proporciona conforto, calor humano e transmite-se a mensagem de que o paciente não esta só diante da dor e do sofrimento.

A pele é o órgão de transformação de estímulos físicos em comunicadores Químicos e em estados psicológicos. Em QualQuer época da vida, um contato terno e amoroso na pele produz a sensação de apoio, consolo, companhia e presença amiga; um contato rude e agressivo faz a pessoa sentir-se rejeitada, desprezada, invadida e provoca-lhe reação de defesa ou raiva ${ }^{(10)}$. O toque caracteriza-se, portanto, como uma forma de comunicação não verbal entre o cliente e o enfermeiro, onde é possível estabelecer empatia na relação, dependendo do sentimento exteriorizado nessa atitude, indo ao encontro de Beck ${ }^{(3)}$, Quando essa diz Que a principal ferramenta de comunicação entre o enfermeiro e o paciente é a empatia, podendo o profissional alcançá-la observando as reações Que provoca nas outras pessoas e também refletindo sobre suas experiências, sendo essa relação, enfermeiro/paciente, a própria essência do propósito da enfermagem.

Essa interação se refere a QualQuer contato durante o Qual dois indivíduos têm influência recíproca se comunicando verbalmente ou não, sendo Que a forma não verbal abrange manifestações tais como a expressão facial, o andar, a postura, o tom de voz, gemidos e gestos $^{(1)}$, configurando a comunicação como o processo que pode capacitar o enfermeiro a estabelecer contato, com a finalidade de ajudar no enfrentamento da doença, do sofrimento e, se necessário, dar assistência para Que seja buscado um significado nessa experiência $^{(3)}$.

Silva ${ }^{(12)}$ já entendia Que são Quatro as funções básicas da 
comunicação não-verbal dentro das relações interpessoais. Inicialmente, seria uma forma de complemento da comunicação verbal, ou ainda uma maneira de substituir a mesma, pode ser também uma contradição ao verbal e por fim uma forma de demonstrar sentimentos.

Em um estudo realizado em um hospital na região norte do estado do Paraná por acadêmicas de enfermagem do $4^{\circ}$ ano, percebeu-se Que $100 \%$ dos enfermeiros utilizavam o toque afetivo e $93 \%$ em pacientes Que precisam de apoio ou consolo; em relação aos pacientes, $86 \%$ vêem o toQue como uma experiência positiva Quando tem necessidade de apoio, $66 \%$ preferem ser tocados nas mãos e $53 \%$ vêem o toque como experiência negativa Quando é técnico e sem afetividade.

O toque esta inserido, portanto, no contexto de manifestações não verbais Que possibilita a enfermagem demonstrarem tanto sua habilidade técnica Quanto sua capacidade de ser solidária e compreensiva $^{(5,7)}$.

\section{METODOLOGIA}

A metodologia utilizada foi analítico-descritiva com abordagem Qualitativa, sendo o presente estudo desenvolvido em duas instituições hospitalares de médio porte em uma cidade no sul do Brasil. Foram sujeitos do estudo sete Enfermeiros identificados com letras do alfabeto grego, a fim de preservar o anonimato. O critério para seleção dos sujeitos foi ser graduado em Enfermagem, atuar em Hospitais Gerais, aceitar Que a entrevista fosse gravada, mostrar interesse em participar do estudo, permitir que os dados analisados fossem divulgados em meio acadêmico e publicados em revista científica.

Para a coleta de dados foram utilizadas entrevistas semiestruturadas $^{(8)}$, as Quais foram gravadas para posterior análise 9 sendo o conteúdo organizado em temas de acordo com o objetivo do estudo e contrastados com a bibliografia consultada e a vivência das peseuisadoras.

Por se tratar de estudo Que envolve seres humanos, as autoras atenderam aos pressupostos da Portaria 196/96 do Conselho Nacional de Saúde; utilizando-se para tanto consentimento livre e esclarecido.

\section{RESULTADOS E DISCUSSÃO}

Para sistematizar os resultados desse estudo, organizamos as falas em duas categorias:

\section{Toque como Ferramenta para estabelecer o "Rapport"}

Conforme foi possível observar, a maioria das entrevistadas declarou tocar afetivamente em seus pacientes, considerando o toque como "[...] abertura de caminho para o relacionamento [...]" (Alfa), indo ao encontro dos pressupostos de Travelbee apud Leopardi ${ }^{(13)}$, a Qual é afirmada Que a relação paciente/enfermeiro é a essência do propósito da enfermagem.

Para Beck $^{(3)}$ a comunicação pode capacitar o enfermeiro a estabelecer uma relação a Qual resultará no processo de interação Que tem como foco "[...] o estabelecimento das relações interpessoais como possibilidade de crescimento para todos os envolvidos, onde os indivíduos exercem influências entre si através da comunicação verbal e não-verbal”.

Armendaris ${ }^{(14)}$ percebeu, em um estudo realizado com profissionais da enfermagem, uma superficialidade na comunicação efetiva, gerando dificuldades na interação equipe/paciente; a comunicação é parte do cuidar, sendo capacidade ou competência interpessoal a ser adQuirida, não importando sua área de atuação, cabendo ao profissional de saúde desenvolver meios, instrumentos e técnicas para oferecer ao paciente uma oportunidade de uma existência mais digna, mais compreensiva e menos solitária ${ }^{(15)}$.

Assim, a comunicação eficiente pode ser vista como recurso terapêutico no cuidado de enfermagem, onde a equipe poderá utilizar esse recurso para diminuir a distância entre o trabalhador em saúde e o cliente, oferecendo desta forma uma assistência eficaz onde o usuário recebe um cuidado Que promova a sua saúde e o valorize eneuanto ser humano ${ }^{(16)}$.

Demonstrações de carinho e o toque terapêutico quando utilizados pelos profissionais, aliviam o sofrimento do paciente, reduz sua ansiedade e possibilita uma interação efetiva e fundamental para o desenvolvimento do cuidado individualizado e voltado para o bem estar do cliente ${ }^{(13)}$.

Através do toque de uma forma expressiva, genuína e sincera, os enfermeiros podem claramente transmitir cuidados e apoio aos clientes e às suas famílias; compreendendo o poder do toque em interações, os enfermeiros podem inseri-lo com sucesso em suas assistências e desenvolver suas próprias habilidades, incluindo-o em processos de comunicação.

Entretanto, observou-se Que um dos sujeitos de estudo relatou "[...] não sentir-se à vontade [...]" (Gama) tocando afetivamente nos pacientes sem antes estabelecer uma relação simpática e empática, o Que demanda um tempo maior de convivência e, conseqüentemente, de internação, o que é mais difícil em uma clínica cirúrgica, ambiente de trabalho da enfermeira em Questão. Essa abordagem é trabalhada por Travelbee apud Leopardi ${ }^{(13)}$ Quando enfoca as fases necessárias para chegar ao Rapport que são: a Fase do encontro original (primeiro contato), Fase das identidades emergentes (interação pessoa/pessoa), Fase da empatia (desejo de estabelecer ajuda mútua), Fase da simpatia (enfermeiro como apoiador para ajudar o paciente a enfrentar a doença e tratamento). Para alcançar o objetivo almejado (fase do Rapport), é demandado certo espaço de tempo para Que se estabeleça a relação, não esquecendo as necessidades do Ser Enfermeiro, Que passa pelas já citadas fases assim como o paciente. Assim é possível entender porque é tão difícil para essa enfermeira tocar afetivamente seus pacientes, pois esses passam sob seus cuidados por poucas horas, não sendo possível estabelecer uma relação interpessoal, Que para ela é indispensável para que possa exercer o toque afetivo. Entretanto, não devemos esquecer que justamente esse cliente no período pós-cirúrgico encontra-se mais fragilizado física e emocionalmente, carente, portanto do toque afetivo; em um estudo realizado com pacientes pós cirúrgicos em uma UTI demonstrou Que o toque tem um significado Que envolve essencialmente o aspecto afetivo, como a segurança, o conforto, a traneüilidade, a compreensão, o olhar com interesse, o encorajamento, enfim, uma forma terapêutica Que capacita o profissional a cuidar do paciente e familiar de forma consciente e humana ${ }^{(17)}$.

Assim, Boff( ${ }^{(18)}$ afirma Que o toque representa o modo de ser cuidado, pois é uma mão revestida de paciência tocando sem agredir, permitindo a mobilidade do ser com Quem entramos em contato. Assim, a mão de Quem toca torna-se algo além do Que uma simples mão, pois no 
estabelecimento de relações transmite Quietude, carinho tocando assim o eu profundo e não apenas a superfície da consciência. Concordamos com Thomas e Carvalho ${ }^{(1 \mathrm{II})} \mathrm{O}$ Qual afirma Que para uma relação enfermeiro/paciente acontecer é necessário a predisposição dos dois seres humanos à concretizá-la, sendo, portanto, indispensável Que a enfermeira Queira realmente tentar este contato, Que poderá ser breve, como um aperto de mão ao desejar um pronto restabelecimento à esse paciente.

\section{Toque como Subsídio para Procedimentos Invasivos}

A execução de procedimentos invasivos é uma das atribuições técnicas da enfermagem Que pressupõe um relacionamento mais próximo com o paciente; sendo essa afirmação comprovada pelas falas das enfermeiras entrevistadas:

\section{"[...] tu amenizas o procedimento com esse toque [...]"(Alfa).}

"[...] tu vai pra realizar um procedimento, um curativo [...] o ideal é tu estabelecer uma afinidade com o paciente pelo toque [...]" (Beta)

"[...] pacientes que ficam mais tempo requerem também mais cuidados, mais procedimentos, nesses sim dá pra fazer [...]" (Gama)

Noda ${ }^{(19)}$, analisando os tipos de toque em pós-operatório, percebeu Que em $54,7 \%$ das interações entre profissional e cliente o toque esteve presente, sendo 58,6\% de toques (aquele Que existe em função da execução de procedimentos ou técnica), 23,9\% foram toques expressivos (aquele Que demonstra atenção, interesse ou sentimento) e em 17,4\% das interações ocorreram ambos os toques (instrumental e expressivo).
Entretanto, percebe-se a realidade da enfermagem como algo mais abrangente do que somente realizar procedimentos invasivos, apesar de Vila e Rossi(1) já haverem estudado sentimentos semelhantes em uma UTI e constatado Que o cuidar pode se tornar "[...] tecnicista e mecânico, desprovido de sentimento." Acreditamos Que o cuidar deve abranger "[...] todo o ser bio-psico-social-espiritual [... ${ }^{\prime(6)}$, levando em consideração suas necessidades afetivas Que emergem em momentos diversos, não somente durante a realização de procedimentos invasivos. Assim como Tomas e Carvalho ${ }^{(11)}$ acredita-se na necessidade de sistematizar uma assistência de enfermagem voltada a atender, além das necessidades biológicas, as necessidades emocionais, psicológicas e espirituais do cliente.

\section{CONSIDERAÇÕES FINAIS}

Foi possível constatar Que, apesar do movimento em prol da humanização no cuidado, as enfermeiras ainda sentem constrangimento ao cuidar do ser holístico, integral, mantendo-se restritas ao cuidado tecnicista exigido. Entretanto salienta-se que há uma preocupação em tornar esse momento basicamente instrumental em um momento mais ameno para o paciente, podendo ser essa atitude considerada uma sinalização para uma gradual evolução ao cuidado humanizado.

Como resultado, observou-se Que as enfermeiras entrevistadas utilizam o toque afetivo de maneira restrita, ou seja, utilizam essa ferramenta de comunicação afetiva não-verbal principalmente durante os procedimentos invasivos a fim de amenizá-los, mesmo considerando-o um bom instrumento para estabelecer a empatia ou Rapport. No nosso entender, o assunto não se esgota com esse trabalho, sendo necessário uma pesQuisa mais ampla, tanto no número de sujeitos como no sentido de averiguar também a visão do paciente relativo à essa Questão.

\section{REFERÊNCIAS}

1. Vila VSC, Rossi LA. O significado cultural do cuidado humanizado em unidade de terapia intensiva: muito falado e pouco vivido. Rev Latino-Am Enfermagem 2002; 10(2): 137-44.

2. Ferreira $\mathrm{ABH}$. Miniaurélio: o minidicionário da língua portuguesa. Curitiba: Positivo; 2004.

3. Beck CLC. Sofrimento e esperança - vivências com familiares de pacientes internados em UTI. In: Gonzales RMB, Beck CLC, Denardin ML. Cenários de cuidado: aplicação das teorias de enfermagem. Santa Maria: Pallotti; 1999. p. 61-157.

4. Siqueira ARP, Cruz ICF. Produção científica de enfermagem sobre o toque: implicações para a (o) enfermeira (o)de cuidados intensivos. [citado em: 26 jun 2005]. Disponível em: http://www.uff.br/nepae/toque.doc

5. Waldow VR. O cuidado humano: o resgate necessário. Porto Alegre: Sagra Luzzatto; 2001 .

6. George JB. Medeleine Leninger. In: George JB. Teorias de enfermagem: os fundamentos à prática profissional . Porto Alegre: Artmed; 2000. p.297-311.

7. Daniel LF. Atitudes interpessoais em enfermagem. São

Paulo: EPU; 1983.

8. Minayo MCS. Pesquisa social. $3^{\mathrm{a}}$ ed. Petrópolis: Vozes; 1994.

9. Chizzoti A. Pesquisa em ciências humanas e sociais. São Paulo: Cortez; 1998.

10. Azambuja R. Tocar faz a diferença. [citado em: 24 jun 2005]. Disponível em: http://www.dermatologia.net/neo/

11. Thomas CT, Carvalho VL. O cuidado ao término de uma caminhada. Santa Maria: Pallotti; 1999.

12. Silva MJP. Comunicação tem remédio: a comunicação nas relações interpessoais em saúde. São Paulo: Editora Gente; 1996.

13. Leopardi MT. Teorias em enfermagem: instrumentos para a prática. Florianópolis: Papa-Livros; 1999.

14. Armendaris M. A comunicação com o paciente no cotidiano da equipe de enfermagem [monografia]. Pelotas (RS): Universidade Federal de Pelotas, Faculdade de Enfermagem; 2003.

15. Stefanelli M. Comunicação com o paciente: teoria e ensino. $2^{\text {a }}$ ed. São Paulo: Robe Editorial; 1993. 
16. Dutra LCR. O processo comucacional em uma instituição hospitalar: um olhar sobre as relações da equipe de enfermagem [dissertação]. Florianópolis (SC): Universidade Federal de Santa Catarina, Programa de PósGraduação em Enfermagem; 2000.

17. Brandão EB, Bastos MRM, Vila VSC. O significado da cirurgia cardíaca e do toque na perspectiva de pacientes internados em UTI. Rev Eletron Enferm 2005; 7(3): 278-84.

18. Boff L. Saber cuidar. $2^{\text {a }}$ ed. São Paulo: Vozes; 1999.

19. Noda KS, Poltronier MIA, Silva MJP. Análise de fatores proxêmicos em situações de pós-operatório. In: Anais do $2^{\circ}$ Congresso Brasileiro de Enfermagem em Centro Cirúrgico. Jul 2005; São Paulo (SP), Brasil. São Paulo: SOBECC; 1995. p. 3-10. 